\section{The pressure phosphene tonometer-a clinical evaluation}

GSM Chew ${ }^{1}$, GF Sanderson ${ }^{2}$ and ACB Molteno ${ }^{2}$

1802, Young ${ }^{2}$ described a bright phantom caused by pressure and in 1823 Purkinje described the subjective impression of a bright central area surrounded by a dark ring with an outer bright halo in some detail. ${ }^{3}$ A pressure phosphene is usually described as a glow, with arcuate or circular characteristics, and is perceived in the visual field opposite to the area of pressure. If the object applying the pressure is small, the centre of the perceived area appears light with a dark surround and a bright outer portion.

However, this appearance is not universal and published descriptions of the phosphenes vary considerably, being described by Helmholtz as 'If the object that exerts the pressure is not large, the phenomenon usually has a bright centre surrounded by a dark ring and by an outer dark one. To the writer it is the brightest when pressure is exerted at or near the equator of the eye where the sclerotica is the thinnest. The pressure-image appears then on the edge of the dark visual field as a bright arc, nearly semicircular in form. ${ }^{3}$ Helmholtz's drawing of his pressure image shows it consisting of a dark spot traversed by a bright vertical band while he points out that 'On the other hand, in a dark visual field there is a bright yellowish circular area within which there is sometimes a dark spot or a dark ring. A dim light is also seen at the entrance of the optic nerve... . ${ }^{3}$ The authors of this paper are unable to appreciate a localised pressure phosphene as described although slightly longer pressure produces kaleidoscopic displays that are discussed by Helmholtz; thus 'Purkinje has studied these phenomena very carefully, and accurately described and represented them. They seem to have had a high degree of regularity for him. The background generally consisted of fine quadrangles in regular array, on which there were either stars with eight rays, or dark or bright rhombs with vertical and horizontal diagonals; and the patterns were surrounded by alternately bright and dark
Pressure phosphenes were known to Aristotle. Mariotte further elaborated on them in $1681 .{ }^{1}$ In
${ }^{1}$ Eye Department

Dunedin Hospital

Dunedin, New Zealand

${ }^{2}$ Section of Ophthalmology Department of Medical and Surgical Sciences University of Otago Dunedin School of Medicine Dunedin, New Zealand

Correspondence:

ACB Molteno

Ophthalmology Section

Department of Medical and Surgical Sciences

University of Otago Dunedin School of Medicine PO Box 913

Dunedin, New Zealand

Tel: +6434747970

Fax: + 6434747628

E-mail: tui.bevin@

stonebow.otago.ac.nz

Received: 11 November 2003

Accepted: 29 March 2004 Published online: 11 June 2004

None of the authors have any proprietary interest in intraocular pressure measuring devices. 
bands. In the author's own experience there is not such regularity in the figures. ${ }^{3}$

The source of pressure phosphenes in the retina is thought to be the bipolar cells, or parts of the rods and cones (photoreceptor nuclei, axons, and terminals), situated anterior to the external limiting membrane. ${ }^{4}$

Pressure phosphene tonometry, based on the entoptic phenomenon of pressure phosphenes, was introduced by Fresco ${ }^{4}$ who has published the only study to date validating the technique by comparing the results of pressure phosphene tonometry with Goldmann applanation tonometry.

Pressure phosphene tonometry is inherently safe, portable, and relatively easy to perform. If reliable, it would be a valuable method for measuring intraocular pressure (IOP). This report compares IOP measurements obtained by pressure phosphene tonometry and Goldmann applanation tonometry in 100 patients attending a general ophthalmology clinic at Dunedin Hospital in 2002.

\section{Materials and methods}

The pressure phosphene tonometer (PPT), Proview ${ }^{\mathrm{TM}}$ eye pressure monitor (Bausch \& Lomb Inc., Tampa, FL, USA), is a spring compression device calibrated in millimetres of mercury and consists of a probe with a flat applicator the same diameter $(3.06 \mathrm{~mm})$ as the area applanated by the Goldmann tonometer (GT). Although the PPT was designed for self-use, the IOPs in this study were measured by the medical staff of the ophthalmology clinic to match the situation in Fresco's original study.

Consecutive patients being assessed in general ophthalmology clinics at Dunedin Hospital in July 2002, who required routine IOP measurement as part of their examination, were approached to participate in this study. Those who consented to participate were informed about the technique and the appearance of the expected phosphene using the artist's impressions in the Bausch \& Lomb Inc. information pamphlet.

Two Proview ${ }^{\mathrm{TM}}$ eye pressure monitors were used by one clinician (GSMC) to perform pressure phosphene tonometry in order to eliminate interobserver variability and instrument error. The PPT was applied to a closed eyelid without topical anaesthetic. The patient was instructed to abduct and depress his/her eye. The PPT was placed on the superior nasal portion of the eyelid. Increasing force was gently applied. Initially, a phosphene was induced with greater force than required in order to familiarise the patient with a phosphene. Subsequently, the patient was instructed to indicate as soon as the pressure phosphene was perceived with the PPT in place. The IOP was read off the graticule. The graticule has a fiduciary indicator that does not move from the highest reading until reset. The use of the superior nasal aspect was chosen as described by Fresco. This area was easy to access and produced a phosphene most readily. This corresponds to the inferotemporal visual field. ${ }^{4}$ In cases where the patient could not perceive a phosphene at the first attempt, the patient was rested for $5 \mathrm{~min}$ and after checking that the explanation had been clearly understood the PPT was applied again. This process was repeated for a total of five attempts before deciding that the patient could not perceive a phosphene. Any error in the PPT readings as a result of the massage effect of repetition would be in the direction of lowering the PPT reading.

Goldmann tonometry was performed by four experienced nurses who were responsible for routine tonometry in ophthalmology clinics using seven different, regularly calibrated Goldmann tonometers mounted on Haag Streit slit lamps. To obtain readings by the GT, one drop of proxymethacaine hydrocholoride $5 \mathrm{mg} / \mathrm{ml}$ was instilled and then a fluorescein strip applied to the palpebral conjunctiva of the lower lid. Fixation was in the primary position of gaze. ${ }^{7}$

Patients were randomised to receive either PPT or GT first and the readings were attempted within $5 \mathrm{~min}$ of each other. There was no communication between the examiners. Results were analysed with reference to Bland and Altman's guidelines. ${ }^{8}$ Ethical approval was obtained from the Otago Ethics Committee.

\section{Results}

In total, 196 eyes of 100 patients were tested. The age range was 21-90 years (median 73 years). GT readings were obtained for all 196 (100\%) eyes. Of the 196 attempted readings, $\mathrm{PPT}$ readings were able to be obtained for $136(69 \%), 68$ (50\%) of whom required more than one attempt to perceive a pressure phosphene. The mean IOPs were $18.5 \mathrm{mmHg}$ (range $8-32 \mathrm{mmHg}$, SD $5.1 \mathrm{mmHg}$ ) using PPT and $16.0 \mathrm{mmHg}$ (range 9$37 \mathrm{mmHg}$, SD $4.3 \mathrm{mmHg}$ ) using GT (Figure 1). Overall, the mean difference (PPT-GT) was $+2.43 \mathrm{mmHg}$; however, there was wide scatter with $95 \%$ confidence intervals ranging from $10.4 \mathrm{mmHg}$ below to $15.2 \mathrm{mmHg}$ above Goldmann readings (Figures 1 and 2). Analysis excluding an outlier (who had recurrence of herptic keratouveitis with corneal oedema but no bullae) with -32 difference between the PPT and GT readings revealed limits of agreement $(95 \% \mathrm{CI})$ of 9.9 below and 15.3 above Goldmann readings.

PPT readings for two eyes were obtained for 59 patients. These observations were not independent in the statistical sense and limits of agreement adjusted for multiple observations for two eyes on the same patient 


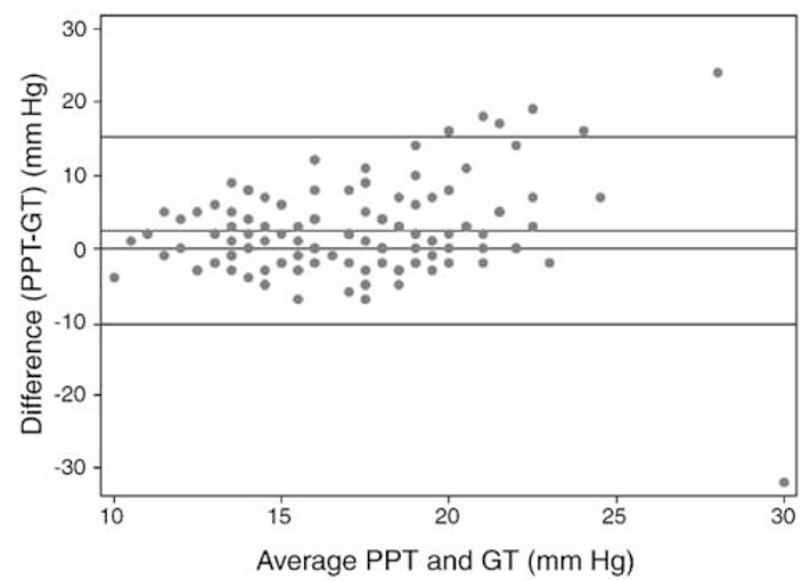

Figure 1 Plot of the difference between PPT and GT readings vs the average PPT and GT readings.

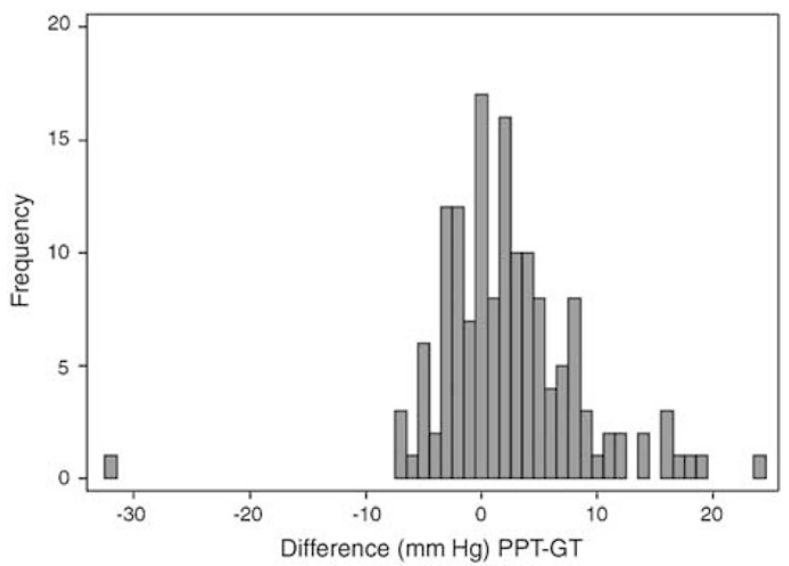

Figure 2 Frequency histogram of the difference between the PPT and GT readings.

were slightly wider at $11.1 \mathrm{mmHg}$ below to $15.7 \mathrm{mmHg}$ above Goldmann readings.

\section{Discussion}

In Fresco's initial report, the mean difference between GT and PPT was $0.3 \mathrm{mmHg}$ below GT in a series of 192 eyes from 100 patients. ${ }^{4}$ However, Fresco's analysis did not take into account inherent statistical dependence when two eyes of a single patient were measured. We have calculated the $95 \%$ confidence interval for mean IOP difference from Fresco's data at $4.3 \mathrm{mmHg}$ below to $4.9 \mathrm{mmHg}$ above Goldmann readings.

In our study, $31 \%$ of patients could not detect a phosphene compared to $3 \%$ of Fresco's patients, ${ }^{4}$ while half of the patients who eventually did perceive a phosphene required more than one attempt to do so. The expected effect of these repeated applications of the PPT before eliciting the phosphene would be to lower the PPT reading and reduce the difference between mean PPT and GT readings. Our data showed a difference between mean IOP readings of $2.43 \mathrm{mmHg}$ above GT with a $95 \%$ confidence interval that the GT reading was between $11.1 \mathrm{mmHg}$ below and $15.7 \mathrm{mmHg}$ above GT. This widespread range indicates that PPT readings cannot be relied on as reliable measures of IOP.

\section{Acknowledgements}

We thank Sheila Williams, biostatistician, Department of Preventative and Social Medicine, Dunedin School of Medicine, for statistical advice; Tui Bevin for help with manuscript revision; and Dr Harry Bradshaw, Margaret O'Connell, Barbara Bucke, Vise Elisara and Vivian Harwood for help with data collection.

\section{References}

1 Duke-Elder S, Gloster J. The stimulus of vision. In: DukeElder S (ed) System of Ophthalmology, Vol IV. Henry Kimpton: London, 1968 pp 447-468.

2 Young T. The Bakerian Lecture. On the mechanism of the eye Phil Trans 1801; 1: 23-89.

3 Southall JPC (ed) Helmholtz's Treatise on Physiological Optics, Vol 2. Dover Publications: New York, 1962 pp 5-7.

4 Fresco BB. A new tonometer-the pressure phosphene tonometer: clinical comparison with Goldmann tonometry. Ophthalmology 1998; 105: 2123-2126.

5 Fresco BB. Pressure phosphene tonometry. Invest Ophthalmol Vis Sci 1997; 38(Suppl): S1056.

6 Goldmann H, Schmidt T. über applanationstonometrie. Ophthalmologica 1957; 134: 221-242.

7 Kass MA. Standardizing the measurement of intraocular pressure for clinical research. Guidelines from the Eye Care Technology Forum. Ophthalmology 1996; 103: 183-185.

8 Bland JM, Altman DG. Statistical methods for assessing agreement between two methods of clinical measurement. Lancet 1986; 8476: 307-311. 